\title{
Application of a 2D Molybdenum Telluride in SERS Detection of Biorelevant Molecules
}

\section{Fraser, James $P$.}

2020-10-21

Fraser , J P , Postnikov , P, Miliutina, E , Kolska, Z, Valiev , R , Svorcik, V , Lyutakov , O , Ganin , A Y \& Guselnikova , O 2020 , ' Application of a 2D Molybdenum Telluride in SERS Detection of Biorelevant Molecules ' , ACS Applied Materials \& Interfaces , vol. 12 , no. 42 , pp. 47774-47783 . https://doi.org/10.1021/acsami.0c11231

http://hdl.handle.net/10138/323374

https://doi.org/10.1021/acsami.0c11231

cc_by

publishedVersion

Downloaded from Helda, University of Helsinki institutional repository.

This is an electronic reprint of the original article.

This reprint may differ from the original in pagination and typographic detail.

Please cite the original version. 


\title{
Application of a 2D Molybdenum Telluride in SERS Detection of Biorelevant Molecules
}

\author{
James P. Fraser, Pavel Postnikov, Elena Miliutina, Zdenka Kolska, Rashid Valiev, Vaclav Švorčík, \\ Oleksiy Lyutakov, Alexey Y. Ganin,* and Olga Guselnikova*
}

Cite This: ACS Appl. Mater. Interfaces 2020, 12, 47774-47783

Read Online

\section{ACCESS I}

Џlll Metrics \& More

Article Recommendations

Supporting Information

ABSTRACT: Two-dimensional (2D) transition-metal dichalcogenides have become promising candidates for surface-enhanced Raman spectroscopy (SERS), but currently very few examples of detection of relevant molecules are available. Herein, we show the detection of the lipophilic disease marker $\beta$-sitosterol on fewlayered $\mathrm{MoTe}_{2}$ films. The chemical vapor deposition (CVD)grown films are capable of nanomolar detection, exceeding the performance of alternative noble-metal surfaces. We confirm that

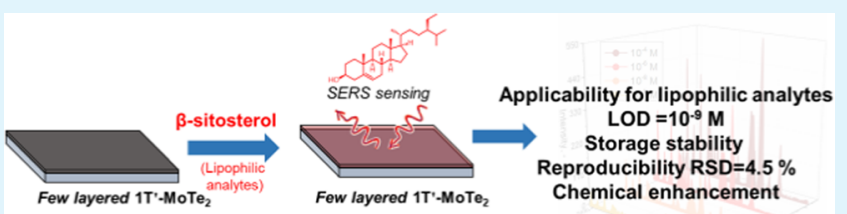
the enhancement occurs through the chemical enhancement (CE) mechanism via formation of a surface-analyte complex, which leads to an enhancement factor of $\approx 10^{4}$, as confirmed by Fourier transform infrared (FTIR), UV-vis, and cyclic voltammetry (CV) analyses and density functional theory (DFT) calculations. Low values of signal deviation over a seven-layered $\mathrm{MoTe}_{2}$ film confirms the homogeneity and reproducibility of the results in comparison to noble-metal substrate analogues. Furthermore, $\beta$-sitosterol detection within cell culture media, a minimal loss of signal over 50 days, and the opportunity for sensor regeneration suggest that $\mathrm{MoTe}_{2}$ can become a promising new SERS platform for biosensing.

KEYWORDS: MoTe, SERS, sensing, chemical enhancement, $\beta$-sitosterol

\section{INTRODUCTION}

Layered two-dimensional (2D) transition-metal dichalcogenides (TMDCs) have emerged as an important class of materials that could bring transformative changes to the ways we fabricate and operate future electronic devices and sensors. ${ }^{1,2}$ Sensing applications, such as surface-enhanced Raman spectroscopy (SERS), rely on strong interactions between an analyte and a surface, making TMDCs particularly well suited for SERS as their atomically flat surfaces facilitate homogeneous distribution of analytes. ${ }^{3}$ Moreover, the ability to fabricate $2 \mathrm{D}$ chalcogenides reproducibly and at scale as fewatom-thick thin films offers an additional benefit for their implementation as stand-alone, noble-metal-free SERS sensing platforms without the need for further functionalization., ${ }^{4,5}$ Previous research has already capitalized on the unique properties of some $2 \mathrm{D}$ materials (such as $\mathrm{MoS}_{2}, \mathrm{MoSe}_{2}$, $\mathrm{WSe}_{2}, \mathrm{NbS}_{2}$, and graphene $)^{6-11}$ to show chemical enhancement (CE) of Raman signals but only with limited successes (low enhancement factors, limited list of analytes, reproducibility issues).

In this regard, the recent demonstration of femtomolar detection of R6G dye on a few-layered 2D semimetal $\mathrm{WTe}_{2}{ }^{7}$ suggests that SERS enhancement in $2 \mathrm{D}$ chalcogenides can at least reach the same order of magnitude as in noble metals. However, in the case of R6G dye, the surface-enhanced resonant Raman scattering (SERRS) phenomenon occurs as excitation laser frequency crosses the frequencies of electronic excited states of the molecule. ${ }^{12,13}$ The SERRS leads to a dramatic increase in the intensity of signal, which means that the use of $2 \mathrm{D}$ materials in practical, real-world applications of clinically relevant molecules, rather than specific dyes, is yet to be discovered to yet be discovered. In this regard, the full potential of TMDCs remains almost unexplored and should be demonstrated using alternative and ideally, relevant nonresonant targeted analytes.

Among clinically relevant molecules, $\beta$-sitosterol stands out as a particularly interesting analyte for investigation as a lipophilic marker of coronary artery disease, cardiovascular disease, and type 1 diabetes. ${ }^{14}$ The detection of $\beta$-sitosterol is complex due to the low level of signal detectable on common noble-metal-based platforms. Previous research showed that grafted gold surfaces with lipophilic functional groups (LFGs) could be utilized for improved sitosterol sensing at $\mathrm{mM}$ concentration. ${ }^{15}$ However, the overlapping Raman peaks of LFGs and $\beta$-sitosterol has limited their application for trace sensing. In this respect, the hydrophobic nature ${ }^{16,17}$ and absence of interfering Raman peaks ${ }^{18,19}$ make metal tellurides

Received: June 20, 2020

Accepted: September 28, 2020

Published: September 28, 2020 


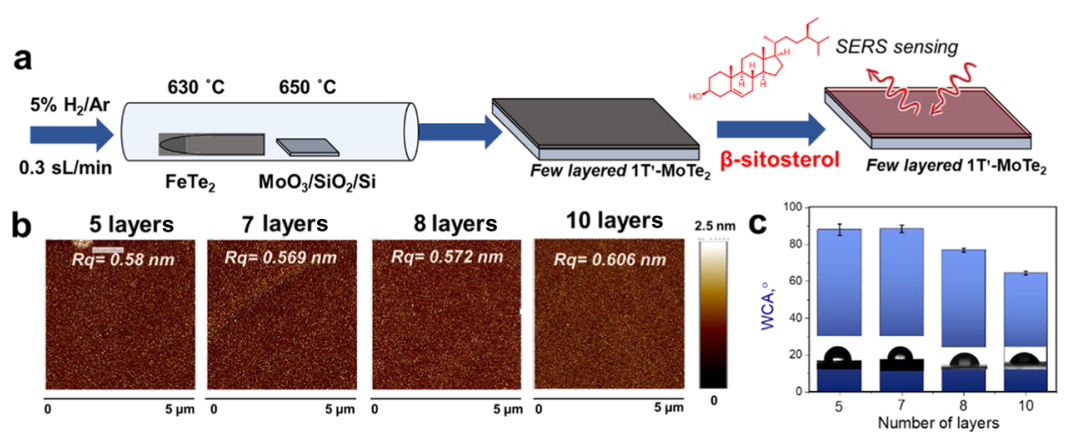

Figure 1. (a) Schematic of a CVD setup used for the synthesis of $\mathrm{MoTe}_{2}$ films and subsequent utilization of prepared 2D films in SERS sensing of $\beta$-sitosterol; (b) atomic force microscopy (AFM) images of metallic $\mathrm{MoTe}_{2}$ films; (c) water contact angle measured on $2 \mathrm{D} \mathrm{MoTe} \mathrm{M}_{2}$ films depending on numbers of layers and the corresponding photographs of water droplets.
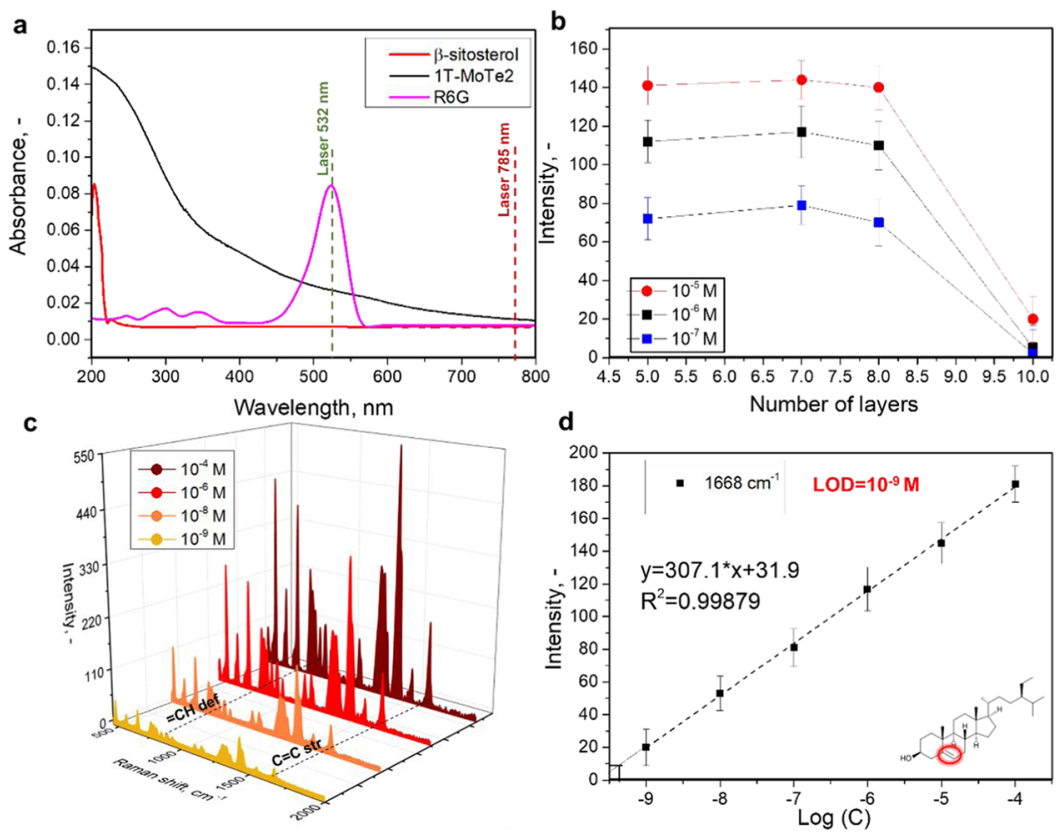

Figure 2. (a) UV-vis spectra of $\beta$-sitosterol, R6G, and $\mathrm{MoTe}_{2}$ film; (b) dependence of SERS peak at $1668 \mathrm{~cm}^{-1}$ measured on $2 \mathrm{D} \mathrm{MoTe}$ films with various numbers of layers; (c) SERS spectra of sitosterol measured on $2 \mathrm{D} \mathrm{MoTe} \mathrm{M}_{2}$ films (seven layers) after deposition at $10^{-4}, 10^{-6}, 10^{-8}$, and $10^{-9} \mathrm{M}$ concentrations on seven-layered $\mathrm{MoTe}_{2}$ surface; and (d) dependence of intensity of two representative SERS peaks $\left(1668 \mathrm{~cm}^{-1}\right)$ on $\beta$ sitosterol concentration.

particularly appealing as sensing platforms toward the detection of $\beta$-sitosterol at trace levels. If proven successful, this could bring $2 \mathrm{D}$ materials a step closer to practical applications and challenge the dominance of noble-metal platforms in SERS bioanalysis.

Herein, we present the first experimental attempt to understand the biosensing properties of semimetallic $\mathrm{MoTe}_{2}$ as a surface plasmon resonance (SPR)-free substrate using $\beta$ sitosterol as an analyte. We demonstrate that $\mathrm{MoTe}_{2}$ films grown by chemical vapor deposition (CVD) show SERS abilities toward $\beta$-sitosterol depending on the number of monolayers within the films. The investigation goes beyond a simple demonstration of the applicability of $\mathrm{MoTe}_{2}$ in SERS of a clinically relevant molecule, as the enhancement mechanism is elucidated, which suggests that the chemical enhancement is primarily driven by the formation of a surface complex.

\section{RESULTS AND DISCUSSION}

Preparation of $1 \mathrm{~T}^{\prime}-\mathrm{MoTe}_{2}$ and Surface Characterization. Utilizing $\beta$-sitosterol as a lipophilic molecule of interest requires a comprehensive assessment of various factors (laser wavelength, enhancement factor, detection limit, signal reproducibility across several sensing experiments, stability over time, sensing ability in the presence of biological matrix as well as explanation for the possible mechanism of signal enhancement), which we carefully investigated in this study. First, $\mathrm{MoTe}_{2}$ thin films were prepared in a horizontal CVD reactor (Figure 1a and Experimental part) by conversion of physically deposited $\mathrm{MoO}_{3}$ on $\mathrm{SiO}_{2} / \mathrm{Si}$ substrates. ${ }^{18}$ The Raman spectra of the films display several intense peaks in the range of $100-270 \mathrm{~cm}^{-1}$, which confirm them as singlephase semimetallic, monoclinic $1 \mathrm{~T}^{\prime}-\mathrm{MoTe}_{2}$ (Figure S1). It is noteworthy that characteristic $\mathrm{MoTe}_{2}$-related peaks are downshifted away from the Raman spectral fingerprints associated with functional groups of typical bioorganic molecules (Figure S1). ${ }^{19,20}$ Previous research demonstrated that modification of gold gratings with LFGs allows for sitosterol sensing at millimolar concentration but overlapping of Raman peaks between LFGs and the analyte has limited gold gratings application in trace sensing. ${ }^{15}$ Conversely, the 
absence of peaks in the spectra of $\mathrm{MoTe}_{2}$ can sufficiently improve the spectral resolution and reproducibility. Several films with thicknesses of 3.6, 4.7, 5.7, and $7 \mathrm{~nm}$ according to atomic force microscopy (AFM) (Figures $1 \mathrm{~b}$ and S2), which corresponded to $5,7,8$, and 10 layers, ${ }^{21}$ were selected and initially probed by water contact angle (WCA) studies (Figure 1c).

Five- and seven-layered $\mathrm{MoTe}_{2}$ films demonstrated hydrophobic behavior as evidenced by WCA $\approx 90^{\circ}$ while as the number of layers increases, WCA decreases, which suggests that the thicker films are more hydrophilic. Considering the lipophilic nature of $\beta$-sitosterol, it is expected that a more homogeneous distribution of target molecule will result in enhanced analyte-surface interactions for hydrophobic films. Moreover, higher affinity of $\mathrm{MoTe}_{2}$ to $\beta$-sitosterol molecules will result in their enhanced surface distribution and adsorption, facilitating the better reproducibility of measurement but not effecting the EF value. Since both five- and seven-layered films displayed a similar WCA, we decided to use seven-layered films for further studies due to a better control over the number of $\mathrm{MoTe}_{2}$ layers during the fabrication process.

Effect of Laser Wavelength. To test the role of the laser wavelength we used $10^{-6} \mathrm{M}$ solution of $\beta$-sitosterol spin-coated on seven-layered $\mathrm{MoTe}_{2}$ and a blank $\mathrm{SiO}_{2} / \mathrm{Si}$ substrate (used as a control) with 532 and $785 \mathrm{~nm}$ lasers (Figure S3). In the case of $532 \mathrm{~nm}$ excitation, the Raman spectra of the samples on both pristine $\mathrm{SiO}_{2} / \mathrm{Si}$ and $\mathrm{MoTe}_{2}$ showed wide and noisy peaks. However, switching the laser wavelength to $785 \mathrm{~nm}$ led to an improvement of the signal with peaks at 900 and 1600 $\mathrm{cm}^{-1}$ recognizable for the samples on blank $\mathrm{SiO}_{2} / \mathrm{Si}$ which suggested the presence of aromatic groups. Remarkably, at 785 $\mathrm{nm}$ excitation the Raman spectra on $\mathrm{MoTe}_{2}$ showed sharp SERS peaks, which could be assigned to the fingerprint vibrations of $\beta$-sitosterol (Figure S3). The most informative Raman peaks for sitosterol are attributed to $=\mathrm{CH}$ out of plane deformation at $889 \mathrm{~cm}^{-1}$ and $\mathrm{C}=\mathrm{C}$ stretch at $1668 \mathrm{~cm}^{-1}$ (see Figure S3 and discussion in the Supporting Information).

To eliminate the possibility of signal enhancement at 532 and $785 \mathrm{~nm}$ wavelengths due to SERRS, which is manifested in coincidence of the Raman laser frequency and the maximum of compound absorption, ${ }^{22}$ we recorded UV-vis spectra of $\beta$ sitosterol (Figure 2a). The observed maximum absorption is at 200-250 nm. This excludes the possibility that the signal enhancement observed with $785 \mathrm{~nm}$ laser is due to SERRS. Potentially SERRS could be induced using a laser at wavelengths of 200-250 nm. However, using such a short wavelength would lead to the emergence of strong fluorescence which would then mask the Raman peaks. ${ }^{23}$ Furthermore, longer wavelength $(785 \mathrm{~nm})$ lasers are routinely applied for the SERS measurement of organic and biomaterials ${ }^{23,24}$ since it offers a balance between competing factors such as Raman signal intensity, fluorescence, and detector sensitivity.

After selecting the excitation wavelength of $785 \mathrm{~nm}$, we probed the SERS response from 5-, 7-, 8-, and 10-layered $\mathrm{MoTe}_{2}$ films (Figure $2 \mathrm{~b}$ ). We found that both five- and sevenlayered $\mathrm{MoTe}_{2}$ surface showed similar signal while a higher number of layers led to a rapid loss of peak intensity, with the $\beta$-sitosterol peaks almost undistinguishable from the background for 10-layered films. The similar rapid decay of peak intensities with increased number of layers has been observed in other 2D layered materials for the sensing of R6G molecules. $^{25-27}$ This phenomenon has been explained from the point of view of the multilayer Fresnel system, where the increase of layer number leads to the lowering of Raman signal of analyte-coated 2D film. ${ }^{28}$ Alternatively, the thicknessdependent nature of electronic band structures has also been considered $^{29}$ as a contributing factor to the total Raman enhancement as the chemisorption capability of the film changes with thickness.

We also noted a correlation between the SERS and the WCA depending on the number of layers (Figure 1c). The interaction between the analyte and the surface plays an important role in the SERS. ${ }^{2,30,31}$ For example, recent research has shown that WCA values of $85-90^{\circ}$ are optimal for the detection of sitosterol. ${ }^{15}$ Therefore, we hypothesized that the higher affinity of $\beta$-sitosterol toward a hydrophobic surface would result in stronger interaction between the $\beta$-sitosterol and film surface.

Furthermore, we calculated the enhancement factor (EF) for the surface as $1.3 \pm 0.25 \times 10^{4}$ (for $1668 \mathrm{~cm}^{-1}$ peak) and $1.0 \pm$ $0.2 \times 10^{4}$ (for $889 \mathrm{~cm}^{-1}$ peak) from the Raman measurement of an equal amount of molecules in the laser spot. The values are lower than previously reported for R6G using a $532 \mathrm{~nm}$ laser, ${ }^{7}$ but as mentioned earlier at the studied wavelength of $785 \mathrm{~nm}$, there is no additional amplification from SERRS in the case of sitosterol. As evident from Figure S4, which shows the spectrum of R6G deposited on a seven-layered $\mathrm{MoTe}_{2}$ film recorded with the $785 \mathrm{~nm}$ laser, once SERRS is removed, a significantly lower EF $=20.5$ (compared to the one recorded using $532 \mathrm{~nm}$ laser) is observed independently of thickness. This is probably due to the poor realization of the CE mechanism and the more hydrophilic nature of the dye. These results are mostly consistent with recently published data on R6G sensing in tellurides.

Surface Complex between $1 \mathrm{~T}^{\prime}-\mathrm{MoTe}_{2}$ and $\boldsymbol{\beta}$-Sitosterol. As we assume the CE mechanism of Raman enhancement, we attempted to identify and characterize any surface complex formed between $\mathrm{MoTe}_{2}$ and sitosterol, which could play a crucial role in the observed sensing performance. ${ }^{32,33}$ The additional effect contributing to SERS is probably CE also known as the first-layer effect. ${ }^{29}$ Its origin can be ascribed to specific interactions, i.e., electronic coupling between molecule and metal, leading to the formation of a surface moleculemetal complex, followed by an increase of the Raman cross section of the adsorbed molecule within this complex. ${ }^{28}$ The formation of a surface complex was proved by appropriate changes of $\zeta$-potential, UV-vis, and Fourier transform infrared (FTIR) spectra, as well as cyclic voltammetry (CV) (see the Supporting Information for a detailed discussion).

According to $\zeta$-potential measurements before the deposition of $\beta$-sitosterol, the surface of $\mathrm{MoTe}_{2}$ is positively charged. Deposition of $\beta$-sitosterol makes the surface negatively charged due to the presence of hydroxy groups ${ }^{34}$ (Figure S5), which may indicate the formation of a surface complex, causing a related increase of Raman scattering cross section and SERS enhancement.

In the UV-vis spectra after sitosterol deposition, we observed the appearance of two low-intensity absorption bands (580 and $210 \mathrm{~nm}$ ) and the most intense absorption bands appear around $910 \mathrm{~nm}$ (Figure S6 and discussion). According to FTIR, after deposition of sitosterol on $\mathrm{MoTe}_{2}$, the spectral position related to $\mathrm{C}-\mathrm{OH}$ is shifted and changed in intensity, suggesting that sitosterol is facing the substrate with polar $\mathrm{OH}$ groups (Figure S7, Table S1, and discussion). 

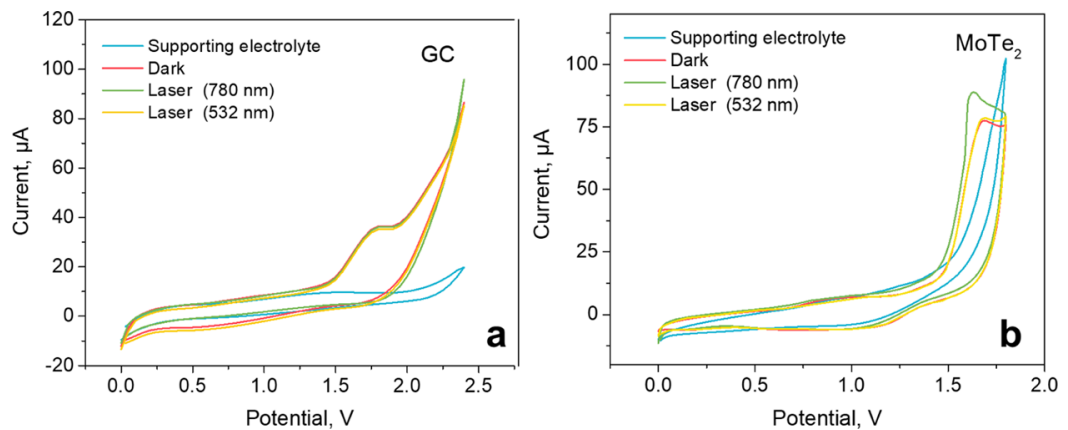

Figure 3. (a) CV scans measured on graphite and (b) $1 \mathrm{~T}^{\prime}-\mathrm{MoTe}_{2}$ in ethanol solution of sitosterol $\left(0.1 \mathrm{M} \mathrm{NaClO}_{4}\right)$ with and without illumination by $780 \mathrm{~nm}$ light source. All potentials are versus $\mathrm{Ag} / \mathrm{AgCl}$ reference electrode.
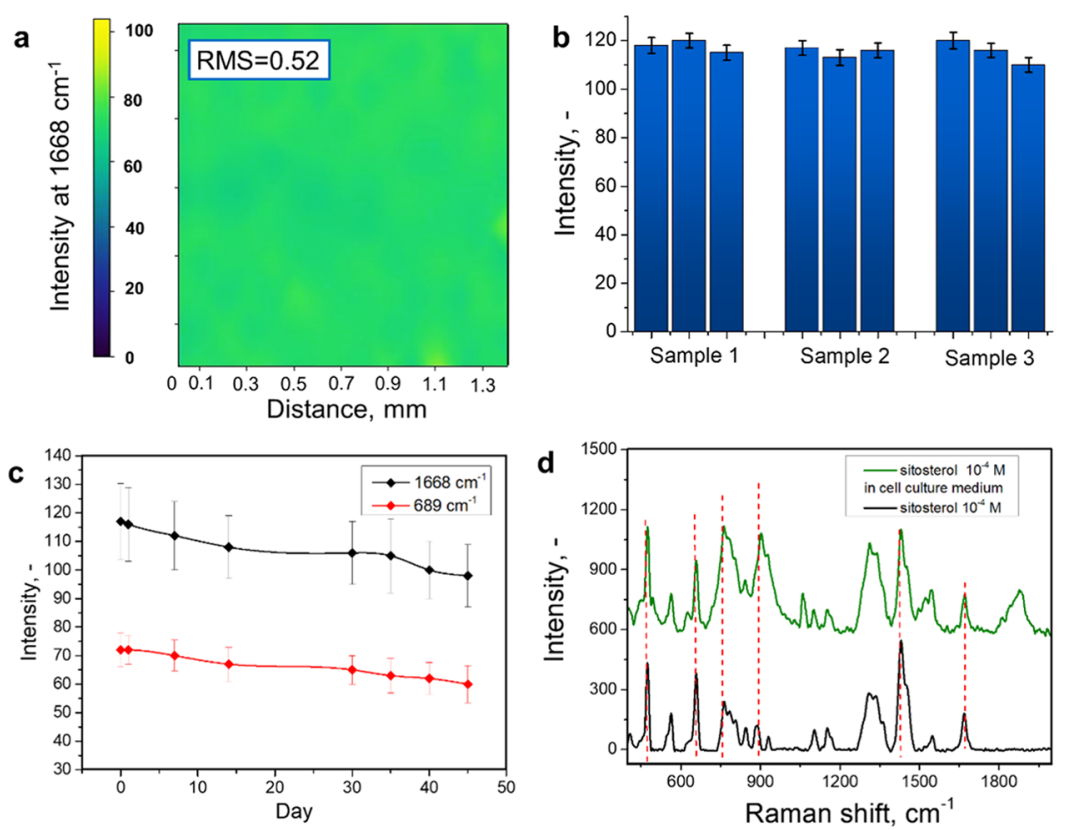

Figure 4. (a) SERS mapping image of $1668 \mathrm{~cm}^{-1}$ peak intensity on seven-layered $\mathrm{MoTe}_{2}$; (b) reproducibility test: variation of $1668 \mathrm{~cm}{ }^{-1}$ peak intensity between the different samples (scale bar presents peak intensity); (c) stability of the $\mathrm{MoTe}_{2}$ determined as the change in intensities of peaks at 1668 and $889 \mathrm{~cm}^{-1}$ with time; and (d) SERS detection of sitosterol in the presence of cell culture medium.

CV scans of sitosterol on graphite (as a control substrate) and $\mathrm{MoTe}_{2}$ (which is robust toward CV measurements) ${ }^{35}$ demonstrate a notable shift of oxidation potential $E_{\mathrm{ox}}$. An increase of peaks on $\mathrm{MoTe}_{2}$ in comparison to graphite indicates that the electrochemical reaction on $\mathrm{MoTe}_{2}$ is due to the enhanced adsorption and surface complex formation (Figure S8 and discussion). We also probed the CV changes during illumination with 780 and $530 \mathrm{~nm}$ wavelengths used for SERS in the previous section (Figure 3).

On a control substrate, the position and intensity of $E_{\mathrm{ox}}$ remain unchanged in the dark and under illumination with both wavelengths. However, there is a significant change of $E_{\mathrm{ox}}$ from 1.67 to $1.58 \mathrm{~V}$ of sitosterol under $780 \mathrm{~nm}$ laser irradiation, also accompanied by an increase in peak intensity under illumination. Conversely, illumination with a $530 \mathrm{~nm}$ laser did not lead to any shift of $E_{\text {ox }}$ and only a negligible increase in peak intensity was observed (Figure 3 and Supporting Information). The results suggest that Raman measurements with the $785 \mathrm{~nm}$ laser facilitates more active electron and charge transfer processes in the sitosterol- $\mathrm{MoTe}_{2}$ complex under illumination. Therefore, this wavelength was utilized in further experiments.
SERS Performance: Evaluation of LOD, Reproducibility, Stability, Selectivity, and Recyclability. Figure $2 c$ shows the SERS spectra of $\beta$-sitosterol deposited on a sevenlayered $\mathrm{MoTe}_{2}$ film for $10^{-4}$ to $10^{-9} \mathrm{M}$ solutions. The characteristic peak intensities (at 1668 and $889 \mathrm{~cm}^{-1}$ ) correlate linearly with the concentration logarithm (Figure 2d), which shows a perfect correlation evident from the correlation coefficients of $R^{2}=0.9988$ (Figures $2 \mathrm{~d}$ and S9). To the best of our knowledge, the calculated limit of detection (LOD) at $10^{-9}$ (based on the minimally acceptable signal intensity; ${ }^{36}$ see the Supporting Information for discussion) is one of the lowest reported so far for SERS detection of sterol-like lipophilic molecules $^{15}$ (see comparative Table S2).

After establishing the LOD for $\beta$-sitosterol, we studied the reproducibility of SERS sensing over the film surface, which is an important factor of SERS in real clinical applications. This can be gauged by the homogeneity of sitosterol coating and verified by SERS mapping ${ }^{37}$ (Figure $4 a$ ). Figure 4 presents a variation of characteristic SERS peak intensity over an area of $1.4 \times 0.8 \mathrm{~mm}^{2}$. A SERS map on $\mathrm{MoTe}_{2}$ shows a very homogeneous distribution of characteristic band intensity along the mapped area (Figure 4a). Standard deviation of 

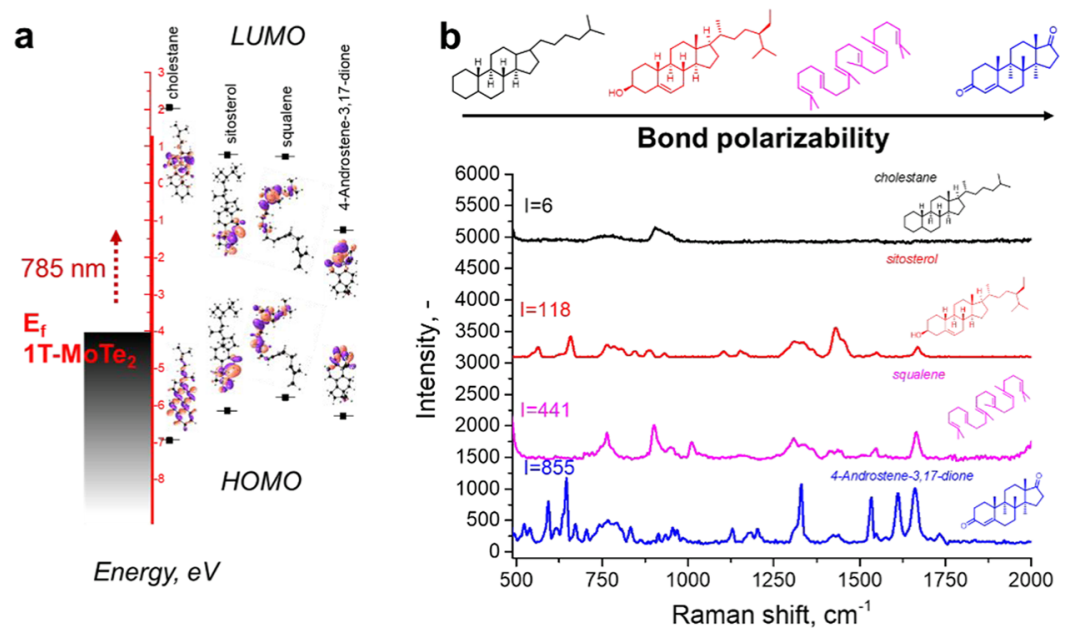

Figure 5. (a) Schematic illustration of the energy band diagrams of $1 \mathrm{~T}^{\prime}-\mathrm{MoTe}_{2}$ and HOMO-LUMO levels of cholestane, sitosterol, squalene, and 4-androstene-3,17-dione. (b) SERS spectra of a series of analytes $\left(10^{-6} \mathrm{M}\right)$ measured on seven-layered $1 \mathrm{~T}^{\prime}-\mathrm{MoTe}_{2}$ with $785 \mathrm{~nm}$ laser.

Raman signal irregularities calculated from the SERS map $\left(1668 \mathrm{~cm}^{-1}\right.$ peak) as mean-square roughness was found to be 0.52 (absolute peak intensity is 117), which demonstrates excellent homogeneity. We also compared the SERS map measured from $\beta$-sitosterol $\left(10^{-6} \mathrm{M}\right)$ deposited on 7 - and 10layered $\mathrm{MoTe}_{2}$ films. We found that the mean-square roughness was 0.52 in the case of seven-layered $\mathrm{MoTe}_{2}$, while utilization of 10 -layered $\mathrm{MoTe}_{2}$ leads to an increase in RMS of almost 10 times, revealing the limited homogeneity of analyte distribution (Figure S10). Apart from the already discussed surface-analyte interaction, the uniform spreading of the analyte could also be attributed to the low roughness of the $\mathrm{MoTe}_{2}$ films (Figure 1b). The repeated homogeneity of the films morphology significantly enhances detection reliability as was evident by the relative standard deviation (RSD) value, which was found to be $4.5 \%$ (number of samples = 3) (see the Supporting Information for calculation details). These values are comparable to RSD 5-6\% reported for common noblemetal sensors, ${ }^{15,38}$ concluding that high reproducibility (a key requirement for modern SERS sensors) is achievable on tellurides (Figure 4b).

The stability of sensing surfaces is another important factor for analytical and clinical applications of SERS. ${ }^{24,39}$ Figure $4 c$ shows the change in the intensity of the Raman peaks of $\beta$ sitosterol $\left(1668\right.$ and $889 \mathrm{~cm}^{-1}$ ) over the period of 50 days storage with the deposited analyte. The signal decreases gradually with an overall loss of less than $15 \%$ over the studied period (see the Supporting Information for discussion).

While the study so far has shown that $\mathrm{MoTe}_{2}$ is applicable for the sensing of $\beta$-sitosterol in model solutions, it is important to test the sensing abilities in the presence of a biological matrix, which can significantly complicate the SERS detection procedure. Therefore, we used $\beta$-sitosterol/fetal bovine serum (FBS) cell culture medium, which is commonly used during studies of the interaction between cell and diseasecausing agents or markers. ${ }^{40,41}$ Freshly spin-coated $10^{-4} \mathrm{M}$ solution of sitosterol in FBS (Dulbecco's modified Eagle's medium) still shows well-distinguishable SERS spectra (Figure 4d). Although there are some overlaps with wide peaks originating from the culture medium, the fingerprint peaks of $\beta$-sitosterol are easily recognized. Remarkably, the utilized biomatrix contains a range of biomolecules in higher concentration than $\beta$-sitosterol, yet it is the peaks of $\beta$ - sitosterol that are predominantly enhanced. The selectivity of the $\mathrm{MoTe}_{2}$ toward $\beta$-sitosterol indirectly confirms the chemical enhancement (CE) mechanism of SERS.

Further, we addressed the possibility of regeneration of the $1 \mathrm{~T}^{\prime}-\mathrm{MoTe}_{2}$ films after sitosterol sensing. The regeneration of a SERS sensor without the loss of functionality is important for various applications. ${ }^{37}$ The results of several cycles of substrate utilization/regeneration are presented in Figure S11. Figure S1la shows the appearance of the characteristic sitosterol SERS bands after the interaction with $10^{-6} \mathrm{M}$ sitosterol and their full disappearance after the substrate regeneration by the immersion and ultrasound $(35 \mathrm{kHz})$ treatment in an ethanol solution for $30 \mathrm{~min}$. One can see that even after five cycles of substrate utilization/regeneration, the mean values of sitosterol characteristic band intensity decrease by only $15 \%$ in comparison to the initial value (Figure S11b). A slight increase in measurement uncertainty is also evident with an increasing number of cycles (measurement errors). Nevertheless, a decrease in the observed value of intensity makes the repeatable use of the proposed substrate possible with a good reproducibility of results and simple preparation and utilization of sensor.

Mechanism of Raman Enhancement. Finally, since the $\mathrm{MoTe}_{2}$ films studied in this work appear as promising noblemetal-free SERS substrates, it is important to discuss the possible Raman signal enhancement mechanism. Commonly, the Raman enhancement can be attributed to the electromagnetic or chemical enhancement (CE) mechanisms. In general, $^{42,43}$ there are three possible scenarios for the $\mathrm{CE}$ mechanism: (1) charge transfer (CT) due to resonance of the incident beam with an excitation from the substrate (a type of the mechanism shown for R6G on $\mathrm{MoTe}_{2}$ ); (2) a molecular resonance mechanism in which the incident beam is resonant with a molecular excitation, and (3) an enhancement due to nonresonant interactions between the surface and the adsorbate. In the third case, the adsorption of the molecule would lead to an increased polarizability and, as a result, an increase of Raman cross section. ${ }^{44}$ To investigate the realization of possible resonance mechanisms (Scenarios 1 and 2), we adopted the calculated band diagram of the $1 \mathrm{~T}^{\prime}$ $\mathrm{MoTe}_{2}$ from our previous work ${ }^{19}$ (Figure 5). $1 \mathrm{~T}^{\prime}-\mathrm{MoTe}_{2}$ is a semimetal with a Fermi level located at $-4.1 \mathrm{eV}$, while the calculated the highest occupied molecular orbital-lowest 
unoccupied molecular orbital (HOMO-LUMO) levels of sitosterol are located at -6.16 and $0.77 \mathrm{eV}$ (Figure 5b) according to the density functional theory (DFT) with B3LYP functional. Unlike R6G (that would resonate with $532 \mathrm{~nm}(2.3$ $\mathrm{eV})^{7}$ due to the HOMO-LUMO band gap of $2.3 \mathrm{eV}$ ), the difference between HOMO-LUMO levels in sitosterol exceeds the excitation wavelength $785 \mathrm{~nm}(1.53 \mathrm{eV})$ or 532 $\mathrm{nm}(2.3 \mathrm{eV})$. Therefore, we excluded the $\mathrm{CE}$ resonance mechanisms.

To probe whether Scenario 3 is responsible for the enhancement we carried out, additional DFT calculations of HOMO-LUMO energies for similar small molecules. Since the difference between HOMO-LUMO levels in sitosterol (Figure 5b) significantly exceeds the excitation wavelength of $785 \mathrm{~nm}(1.53 \mathrm{eV})$, we hypothesized that the enhancement is due to ground-state chemical interactions between the analyte and the surface (i.e., are not associated with the resonance of HOMO-LUMO energies and the excitation wavelength in the free molecule). $\zeta$-potential, FTIR, UV-vis, and CV measurements (see Figures 3 and S5-S8, and the discussion in the Supporting Information) confirm that a surface complex is formed when $\mathrm{MoTe}_{2}$ is used as a substrate. As evident from $\mathrm{CV}$ measurements in Figure 3, the complex has differing energy levels from the free sitosterol molecule, and therefore, the level of interactions between the complex and the Fermi energy level in $1 \mathrm{~T}^{\prime}-\mathrm{MoTe}_{2}$ would be different from what was expected for the free molecule, as shown in Figure 5. Groundstate models rely on the fact that even in the ground state there is a charge transfer between the surface and molecules. ${ }^{45}$ This transfer is modulated by molecular vibrations and thus gives rise to Raman bands. The enhancement in these modes has been attributed to the high-scattering cross section of the metal, which contributes to the intensity of Raman bands from the surface complex. ${ }^{44,45}$ Therefore, based on $\zeta$-potential studies (Figure S5), we hypothesize that a complex is formed due to a surface-dipole interaction. In this case, the Raman enhancement would depend on the ability of the analyte molecule to polarize and to accept $\pi$-backbonding (significant stabilization of the HOMO-LUMO gaps $)^{44}$ upon contact with $\mathrm{MoTe}_{2}$. Polarizability is a dynamic property of the analyte in the presence of external stimuli, which is represented here by the $\mathrm{MoTe}_{2}$ surface.

To test this hypothesis, we probed a range of lipophilic biorelevant molecules with differing polarizabilities (Figure $5 b)$. As can be seen from the energy diagram, the increase of polarizability occurs simultaneously with the decrease of HOMO-LUMO gap of the molecules. The assumed polarizability increases in the following order: cholestane (almost no polarization can be observed due to the presence of $\mathrm{C}-\mathrm{H}$ and $\mathrm{C}-\mathrm{C}$ bonds in structure) $<$ sitosterol $(\mathrm{OH}$ groups and $\mathrm{C}=\mathrm{C}$ bond $)<$ squalene ( $6 \mathrm{C}=\mathrm{C}$ bonds $)<$ andostene-3,17-dione (two $\mathrm{C}=\mathrm{O}$ groups and $\pi-\pi$ conjugation between $\mathrm{C}=\mathrm{O}$ and $\mathrm{C}=\mathrm{C}$ bonds). ${ }^{46}$ All HOMO-LUMO energies were also calculated using DFT. Based on previous work on the CHEM mechanism, ${ }^{44}$ the introduction of acceptor groups with increased polarizability leads to the increase of $\pi$-backbonding. In general, it was demonstrated that CHEM mechanism could lead to an EF in the range 10-1000 by simply changing the functional groups on the analyte. ${ }^{44,47}$ The sensing of cholestane, $\beta$-sitosterol, squalene, and 4-andostene-3,17-dione at $10^{-6} \mathrm{M}$ concentrations on $1 \mathrm{~T}^{\prime}-\mathrm{MoTe}_{2}$ demonstrates the increase of Raman signal intensity (EF could not be compared due to a difference in band enhancement in all molecules) within this range of molecules. In the case of sitosterol, the selective enhancement with $785 \mathrm{~nm}$ could be explained by the fact that (unlike free sitosterol) the surface complex could resonate with $785 \mathrm{~nm}(1.53 \mathrm{eV})$ excitation wavelength. This assumption is supported by the experimental change in $\mathrm{CV}$ scans of sitosterol on $\mathrm{MoTe}_{2}$ under illumination with $780 \mathrm{~nm}$ laser (Figure 3). The results suggest that only the irradiation of the $780 \mathrm{~nm}$ laser during Raman measurements facilitates more active electron and charge transfer processes, revealing a change in the excitation energy of the surface complex formed. $^{48}$

\section{CONCLUSIONS}

In conclusion, we present the first example of SERS biosensing on few-layered $\mathrm{MoTe}_{2}$ films for $\beta$-sitosterol detection, confirming the applicability of $2 \mathrm{D}$ tellurides beyond the sensing of common dye molecules. The relationship between the thickness of CVD-grown films and recorded SERS signal showed that the enhancement factor rapidly diminishes as the number of layers increase, while the impressive performance (down to nanomolar detection level) was achievable even on relatively thick seven-layered films. This implies that extremely low values of detection limit can be realized at scale and highthroughput manner on 2D telluride surfaces. The Raman mapping results with an RSD value of $4.5 \%$ suggested that the smoothness and hydrophobicity of film surfaces facilitated the uniform distribution of analyte, which led to consistency and reproducibility of the results. Furthermore, the distinct ability for $\beta$-sitosterol detection within a complex cell culture media suggests that the sensing ability of $\mathrm{MoTe}_{2}$ expands beyond the simple recognition of model solutions under idealized conditions. The sensor can be easily regenerated for at least five cycles without significant loss of sensing performance. Therefore, we conclude that the simplicity of the fabrication procedure and high reproducibility of the experiments make 2D tellurides viable targets for the future design of SERS platforms capable of detecting clinically relevant molecules. We hypothesize that the sensing of $\beta$-sitosterol is realized via nonresonant charge transfer processes as the formation of charged surface complex was confirmed by $\zeta$-potential measurements, UV-vis, FTIR, and CV as well as by variation of the SERS observed for analytes with variable polarizability. It appears that this complex originates from surface-dipole interaction, Raman enhancement, and governed by the ability of the molecule to polarize in the contact with $\mathrm{MoTe}_{2}$ and readily accept $\pi$-backbonding. However, the detailed computational investigation of the chemical enhancement mechanism, which is required to reveal the crucial role of excitation wavelength, goes beyond the scope of this paper due to the dynamic nature of the complex. The obtained findings reveal the potential applicability of enabling ultrasensitive sensing of lipophilic analytes due to CE mechanism.

\section{EXPERIMENTAL SECTION}

Materials. $\beta$-Sitosterol (primary pharmaceutical reference standard) and phosphate-buffered saline $(10 \times$ concentrate $)$ were purchased from Sigma-Aldrich and used without further purification.

Pure Cell Culture Medium. Dulbecco's modified Eagle's medium (DMEM) supplemented with antibiotics (penicillin $[100 \mathrm{U} / \mathrm{mL}]$ and streptomycin $[100 \mu \mathrm{g} / \mathrm{mL}]$ ) (both Sigma-Aldrich, Prague, Czech Republic) and $10 \%$ of fetal bovine serum (FBS) was divided into 1 $\mathrm{mL}$ aliquots in sterile Eppendorf tubes and stored at $-80{ }^{\circ} \mathrm{C}$. 
Preparation of $\mathrm{MoTe}_{2}$ Films. $\mathrm{SiO}_{2} / \mathrm{Si}(100)$ (Inseto) substrates $\left(10 \times 10 \mathrm{~mm}^{2}, 300 \mathrm{~nm}\right)$ were first cleaned by sonication in acetone for $10 \mathrm{~min}$, followed by sonication in isopropyl alcohol for a further $10 \mathrm{~min}$ before being dried under a stream of nitrogen gas.

Varied-thickness $\mathrm{MoO}_{3}$ films were deposited by thermal evaporation of $\mathrm{MoO}_{3}$ pellets (99.99\%, Pi-Kem) in a Plassys MEB400 under a vacuum of $2 \times 10^{-6} \mathrm{mbar}$ at a rate of $0.2 \mathrm{~nm} / \mathrm{s}$.

$1 \mathrm{~T}^{\prime}-\mathrm{MoTe}_{2}$ films were synthesized via chemical vapor deposition. $\mathrm{MoO}_{3} / \mathrm{SiO}_{2} / \mathrm{Si}$ substrates were placed into the hot zone $\left(650^{\circ} \mathrm{C}\right)$ of a Lenton tube furnace equipped with a quartz work tube (length: 43.5 $\mathrm{cm}$, external diameter: $20 \mathrm{~mm}$, internal diameter: $17 \mathrm{~mm}$ ). The $\mathrm{FeTe}_{2}$ precursor $(33 \mathrm{mg})$ was placed $7 \mathrm{~cm}$ upstream $\left(630^{\circ} \mathrm{C}\right)$ of the $\mathrm{MoO}_{3} /$ $\mathrm{SiO}_{2} / \mathrm{Si}$ substrate. The work tube was connected by Swagelock to a vacuum pump, allowing the system to be evacuated to a pressure of 1 $\times 10^{-1}$ mbar before being refilled with the $5 \% \mathrm{H}_{2} / \mathrm{Ar}$ carrier gas. This process was repeated for three cycles ensuring an oxygen-free environment for the deposition. The tube furnace was ramped to the deposition temperature $\left(650{ }^{\circ} \mathrm{C}\right)$ at a rate of $5{ }^{\circ} \mathrm{C} / \mathrm{min}$ with a dwell time of $4 \mathrm{~h}$ before cooling to room temperature at a rate of 5 ${ }^{\circ} \mathrm{C} / \mathrm{min}$. The flow rate of the carrier gas was maintained at $300 \mathrm{sccm}$ throughout the deposition.

Deposition of Analytes. Analyte solutions of $\beta$-sitosterol and cholestan were prepared by dissolution of solid with different concentrations $\left(10^{-4}, 10^{-5}, 10^{-6}, 10^{-7}, 10^{-8}, 10^{-9} \mathrm{M}\right)$ in water/ methanol (1:2) and spin-coated on sample (300 rpm, $10 \mathrm{~min}$ ).

Characterization. Atomic Force Microscopy. Atomic force microscopy measurements were carried out on a Bruker Dimension Icon AFM using ScanAsyst mode. Surface roughness measurements were obtained from a scan area of $5 \times 5 \mu \mathrm{m}^{2} .1 \mathrm{~T}^{\prime}-\mathrm{MoTe}_{2}$ film thicknesses were measured relative to bare $\mathrm{SiO}_{2} / \mathrm{Si}$ substrate over an area of $3 \times 3 \mu \mathrm{m}^{2}$. To create the step between the $\mathrm{MoTe}_{2}$ film and $\mathrm{SiO}_{2} / \mathrm{Si}$ substrate, a marker pen was used to draw an $\sim 3 \mathrm{~mm}$ thick line across a cleaned substrate prior to $\mathrm{MoO}_{3}$ deposition. Once $\mathrm{MoO}_{3}$ had been deposited, the substrate was sonicated in isopropyl alcohol for $30 \mathrm{~s}$, which dissolved the marker pen, taking a strip of $\mathrm{MoO}_{3}$ with it and leaving a strip of bare $\mathrm{SiO}_{2} / \mathrm{Si}$ next to $\mathrm{MoO}_{3}$. The $\mathrm{MoO}_{3}$ was then converted to $1 \mathrm{~T}^{\prime}-\mathrm{MoTe}_{2}$ using the procedure described above. The thickness of the resulting $1 \mathrm{~T}^{\prime}-\mathrm{MoTe}_{2}$ film could be measured against the bare $\mathrm{SiO}_{2} / \mathrm{Si}$ substrate. A new tip was used for each sample to minimize errors associated with the AFM instrument.

Wettability Measurements. Water CAs were measured by a Drop Shape Analyzer-DSA100 (Kruss, Germany) at 10 positions (drop volume, $2 \mu \mathrm{L}$ ) at room temperature.

Electrokinetic Analysis. Electrokinetic analysis ( $\zeta$-potential) of all samples was performed by SurPASS Instrument (Anton Paar GmbH, AT). The samples were studied inside the adjustable gap cell with an electrolyte of $0.001 \mathrm{M} \mathrm{KCl}$. All samples were measured four times at constant $\mathrm{pH} 6.3$ and room temperature. For $\zeta$-potential determination, the streaming current method and Helmholtz-Smoluchowski (HS) equation were used. The final values of $z$-potential are subtracted from the $\mathrm{SiO}_{2} /$ Si-derived value of $-23.5 \mathrm{mV}$.

Raman Spectra. Raman spectra of $1 \mathrm{~T}^{\prime}-\mathrm{MoTe}_{2}$ were collected in backscattering configuration on a Horiba-JY HR800 spectrometer with a $532 \mathrm{~nm}$ laser excitation wavelength (laser power at sample, 10 $\mathrm{mW}$ ) and a $600 \mathrm{~g} / \mathrm{mm}$ grating. The spectra were acquired using a $50 \times$ objective with a $10 \mathrm{~s}$ accumulation time. Raman spectra of $\beta$-sitosterol on $\mathrm{MoTe}_{2}$ were collected on a ProRaman-L (laser power, $70 \mathrm{~mW}$; excitation wavelength, $785 \mathrm{~nm}$ ) Raman spectrometer. The spectra were measured 100 times, each of them with $1 \mathrm{~s}$ accumulation time. Raman spectra were used for chemical mapping with a surface area of $1.4 \times 0.8 \mathrm{~mm}^{2}$, having $70 \times 12$ points spaced by a gap of $0.05 \mathrm{~mm}$. The same Raman spectrometer fitted with a source shining at $785 \mathrm{~nm}$ and $70 \mathrm{~mW}$ power was used for the mapping. The spectra were recorded at a resolution of $2 \mathrm{~cm}^{-1}$ in the $2000-500 \mathrm{~cm}^{-1}$ wavenumber range with an exposure time of $100 \mathrm{~s}$ for 1 exposure.

Regeneration of Sensor. After the sensing of sitosterol, the $1 \mathrm{~T}^{\prime}$ $\mathrm{MoTe}_{2}$ films were immersed in $5 \mathrm{~mL}$ of ethanol and then treated with ultrasound for $30 \mathrm{~min}(35 \mathrm{kHz}$, SONOPULS mini20) followed by drying in air. The fresh $10^{-6} \mathrm{M}$ solution of sitosterol in water/ methanol (1:2) was applied on a dry substrate.
Fourier Transform Infrared (FTIR)-Attenuated Total Reflection (ATR). Fourier transform infrared (FTIR)-attenuated total reflection (ATR) spectra were recorded using a Nicolet 6700 spectrometer (Thermo Scientific, France) with a Smart ATR accessory device (2000 scans and $4 \mathrm{~cm}^{-1}$ resolution).

UV-Vis Spectra. UV-Vis spectra were measured using a Spectrometer Lambda 25 (PerkinElmer) in $200-1100 \mathrm{~nm}$ wavelength range.

Cyclic Voltammetry. Cyclic voltammetry (CV) measurements were performed with potentiated Palm Sens 4 (Palm Instruments, the Netherlands), controlled by PSTrace 5.3 software (5.3.1127 firmware), within a potential range of $0-2000 \mathrm{mV}$ and a linear potential sweep rate of $100 \mathrm{mV} / \mathrm{s}$ using $\mathrm{Pt}$ as an auxiliary electrode and $\mathrm{Ag} / \mathrm{AgCl}(3 \mathrm{M} \mathrm{KCl})$ as a reference electrode. Before the measurements, the Pt electrode was polished with $0.05-0.01 \mu \mathrm{m}$ $\mathrm{Al}_{2} \mathrm{O}_{3}$ powder on a wet pad and washed in water. The measurement used $5 \mathrm{~mL}$ of the supporting electrolyte consisting of $1 \mathrm{M}$ sitosterol/ $0.1 \mathrm{M} \mathrm{NaClO}_{4}$ solution in ethanol. Light-emitting diode (LED) irradiation was done for 10 min continuously with $780 \mathrm{~nm}, 7 \mu \mathrm{W} /$ $\mathrm{mm}^{2}$, and $530 \mathrm{~nm}, 9 \mu \mathrm{W} / \mathrm{mm}^{2}$ sources by Thorlabs. All electrochemical measurements were repeated three times.

DFT Calculations. The optimization geometry of the ground electronic state of I-IV molecules was performed using the FIREFLY software $^{49}$ at the density functional level of theory (DFT) ${ }^{50}$ with B3LYP functional ${ }^{51} 6-31 G^{* *}$ basis set. ${ }^{52}$ Also, the energies of highest occupied molecular orbital (HOMO) and lowest unoccupied molecular orbital (LOMO) were calculated at the same level of theory. The schematic description of HOMO and LUMO was performed using Chemcraft software. ${ }^{53}$

Calculation of the SERS Enhancement Factor (EF). The sitosterol $10^{-6}$ and $10^{-3} \mathrm{M}$ solutions was spin-coated on the $\mathrm{MoTe}_{2}$ substrates and appropriated control surface (silicon was used as reference substrate) and measured (with preliminary adjustment of analyzed spot for equal number of analyzed molecules) with a laser spot $5 \mu \mathrm{m}$. Calculation of SERS EF was performed according to the standard relation

$$
\begin{aligned}
& \mathrm{EF}=\left(I_{\text {SERS }} / N_{\text {SERS }}\right)\left(I_{\mathrm{RS}} / N_{\mathrm{RS}}\right) \\
& \text { peak at } 1668 \mathrm{~cm}^{-1}: \mathrm{EF} \\
& \quad=\left(117 / 3 \times 10^{-6}\right)\left(3 \times 10^{-3} / 9\right) \\
& \quad=1.3 \times 10^{4} \\
& \text { peak at } 889 \mathrm{~cm}^{-1}: \mathrm{EF} \\
& =\left(73 / 3 \times 10^{-6}\right)\left(3 \times 10^{-3} / 7.1\right) \\
& =1.0 \times 10^{4}
\end{aligned}
$$

where $I_{\mathrm{SERS}}$ and $I_{\mathrm{RS}}$ represent the Raman scattering intensities on SERS-active and reference silicon surfaces $(70 \mathrm{~mW})$, respectively. The analyte solution with $100 \mu \mathrm{L}$ volume was spin-coated on surface with an $\sim 0.5 \mathrm{~cm}^{2}$ substrate area. $N_{\text {SERS }}$ is written as $N_{\text {SERS }}=c V N_{\mathrm{A}} A_{1} / A_{\text {sub }}$, where $c$ is the analyte concentration, $V$ is the dye droplet volume, $N_{\mathrm{A}}$ is the Avogadro constant, $A_{1}$ is the laser spot area, and $A_{\text {sub }}$ is the substrate area.

Calculation of LOD. $N$ Limit of detection was calculated from the signal-to-noise approach, according to the IUPAC recommendation. ${ }^{36}$ In particular, the minimal concentration at which the signal-to-noise relation is equal to 3 was considered as the "limit of detection (LOD)". The standard deviation of background signal (noise) was calculated from 20 spectra of $\mathrm{MoTe}_{2}$ (seven layers) according to the relation $\mathrm{SD}=\sqrt{\frac{\sum|x-\bar{x}|}{N}}$ (where $x$ is the value of $\mathrm{C}=\mathrm{C}$ signal at 1668 $\mathrm{cm}^{-1}, \overline{\mathrm{x}}$ is the mean of the intensities, $N$ is the number of used spectra) and was found to be $\mathrm{SD}=3.2$.

According to the IUPAC recommendation, the minimally acceptable signal intensity must be 3 times greater than the background signal deviation, i.e., the intensity of $\mathrm{C}-\mathrm{S}$-related peak should have an absolute value equal to 9.6. As is depicted in Figure 3b, which presents the linear fit of peak intensity of the sitosterol concentration 
dependency, this intensity approximately corresponded to the $10^{-9.3}$ $\mathrm{M}$ concentration of sitosterol. To provide conference value to the readers, the LOD was established to be $10^{-9} \mathrm{M}$.

\section{ASSOCIATED CONTENT}

\section{s) Supporting Information}

The Supporting Information is available free of charge at https://pubs.acs.org/doi/10.1021/acsami.0c11231.

Raman spectra of $1 \mathrm{~T}^{\prime}-\mathrm{MoTe}_{2}$ films with varying thicknesses; AFM profiles of the interfaces between few-layered $1 \mathrm{~T}^{\prime}-\mathrm{MoTe}_{2}$ films and $\mathrm{SiO}_{2} / \mathrm{Si}$ substrates; SERS spectra of $\beta$-sitosterol $\left(10^{-6} \mathrm{M}\right)$ on $\mathrm{SiO}_{2} / \mathrm{Si}$ and $\mathrm{MoTe}_{2} ;$ SERS spectra of R6G on $\mathrm{MoTe}_{2}$ film; $\zeta$ potential measurements of $\mathrm{MoTe}_{2}$ before and after $\beta$ sitosterol deposition; UV-vis spectra of $\beta$-sitosterol on $\mathrm{MoTe}_{2}$ and sapphire; FTIR spectra of $\beta$-sitosterol on $\mathrm{MoTe}_{2}$ and $\beta$-sitosterol; CV measurements of $\beta$ sitosterol on $\mathrm{MoTe}_{2}$ and $\beta$-sitosterol on graphite; dependence of intensity of representative SERS peak on sitosterol concentration; comparison of various SERS sensors for the detection of lipophilic molecules; SERS mapping of $1668 \mathrm{~cm}^{-1}$ peak intensity on 7- and 10layered $\mathrm{MoTe}_{2}$; assessment of the stability of $\mathrm{MoTe}_{2}$ films; and calculation details of HOMO-LUMO energies for analytes: cholestane, $\beta$-sitosterol, squalene, and 4-andostene-3,17-dione (PDF)

\section{AUTHOR INFORMATION}

\section{Corresponding Authors}

Alexey Y. Ganin - School of Chemistry, University of Glasgow, Glasgow G12 8QQ United Kingdom; 이이.org/00000002-3754-5819; Email: alexey.ganin@glasgow.ac.uk

Olga Guselnikova - Department of Solid State Engineering, University of Chemistry and Technology, 16628 Prague, Czech Republic; Research School of Chemistry and Applied Biomedical Sciences, Tomsk Polytechnic University, Tomsk 634050, Russian

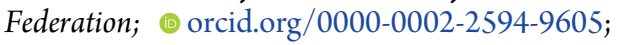

Email: olga.guselnikova@vscht.cz

\section{Authors}

James P. Fraser - School of Chemistry, University of Glasgow, Glasgow G12 8QQ, United Kingdom; @ orcid.org/00000001-7751-4593

Pavel Postnikov - Department of Solid State Engineering, University of Chemistry and Technology, 16628 Prague, Czech Republic; Research School of Chemistry and Applied Biomedical Sciences, Tomsk Polytechnic University, Tomsk 634050, Russian Federation; 이잉.org/0000-0001-9713-1290

Elena Miliutina - Department of Solid State Engineering, University of Chemistry and Technology, 16628 Prague, Czech Republic; Research School of Chemistry and Applied Biomedical Sciences, Tomsk Polytechnic University, Tomsk 634050, Russian Federation

Zdenka Kolska - Faculty of Science, J. E. Purkyne University, 40096 Usti nad Labem, Czech Republic

Rashid Valiev - Department of Solid State Engineering, University of Chemistry and Technology, 16628 Prague, Czech Republic; Department of Chemistry, University of Helsinki, Helsinki FIN-00014, Finland

Vaclav Šrčík - Department of Solid State Engineering, University of Chemistry and Technology, 16628 Prague, Czech Republic
Oleksiy Lyutakov - Department of Solid State Engineering, University of Chemistry and Technology, 16628 Prague, Czech Republic; Research School of Chemistry and Applied Biomedical Sciences, Tomsk Polytechnic University, Tomsk 634050, Russian Federation; (1) orcid.org/0000-0001-8781-9796

Complete contact information is available at: https://pubs.acs.org/10.1021/acsami.0c11231

\section{Notes}

The authors declare no competing financial interest.

\section{ACKNOWLEDGMENTS}

This work was supported by Tomsk Polytechnic University Competitiveness Enhancement Program (VIU-RSCABS-194/ 2020), RFBR 19-43-703002, Grant Agency of Czech Republic ACR 19-24603Y. A.Y.G. and J.P.F. acknowledge the University of Glasgow, EPSRC (EP/P001653/1) and (EP/N509668/1), and the Carnegie Trust for a Research Incentive Grant (RIG007428) for supporting this work.

\section{REFERENCES}

(1) Naumis, G. G.; Barraza-Lopez, S.; Oliva-Leyva, M.; Terrones, H. Electronic and Optical Properties of Strained Graphene and Other Strained 2D Materials: A Review. Rep. Prog. Phys. 2017, 80, No. 096501.

(2) Glavin, N. R.; Rao, R.; Varshney, V.; Bianco, E.; Apte, A.; Roy, A.; Ringe, E.; Ajayan, P. M. Emerging Applications of Elemental 2D Materials. Adv. Mater. 2020, 32, No. 1904302.

(3) Xia, M. 2D Materials-Coated Plasmonic Structures for SERS Applications. Coatings 2018, 8, No. 137.

(4) Kannan, P. K.; Shankar, P.; Blackman, C.; Chung, C. H. Recent Advances in 2D Inorganic Nanomaterials for SERS Sensing. Adv. Mater. 2019, 31, No. 1803432.

(5) Yin, Y.; Miao, P.; Zhang, Y.; Han, J.; Zhang, X.; Gong, Y.; Gu, L.; Xu, C.; Yao, T.; Xu, P.; Wang, Y.; Song, B.; Jin, S. Significantly Increased Raman Enhancement on $\operatorname{MoX}_{2}(X=S$, Se) Monolayers upon Phase Transition. Adv. Funct. Mater. 2017, 27, No. 1606694.

(6) Singha, S. S.; Mondal, S.; Bhattacharya, T. S.; Das, L.; Sen, K.; Satpati, B.; Das, K.; Singha, A. Au Nanoparticles Functionalized 3D$\mathrm{MoS}_{2}$ Nanoflower: An Efficient SERS Matrix for Biomolecule Sensing. Biosens. Bioelectron. 2018, 119, 10-17.

(7) Tao, L.; Chen, K.; Chen, Z.; Cong, C.; Qiu, C.; Chen, J.; Wang, X.; Chen, H.; Yu, T.; Xie, W.; Deng, S.; Xu, J.-B. 1T' Transition Metal Telluride Atomic Layers for Plasmon-Free SERS at Femtomolar Levels. J. Am. Chem. Soc. 2018, 140, 8696-8704.

(8) Tan, Y.; Ma, L.; Gao, Z.; Chen, M.; Chen, F. Two-Dimensional Heterostructure as a Platform for Surface-Enhanced Raman Scattering. Nano Lett. 2017, 17, 2621-2626.

(9) Zheng, Z.; Cong, S.; Gong, W.; Xuan, J.; Li, G.; Lu, W.; Geng, F.; Zhao, Z. Semiconductor SERS Enhancement Enabled by Oxygen Incorporation. Nat. Commun. 2017, 8, No. 1993.

(10) Song, X.; Wang, Y.; Zhao, F.; Li, Q.; Ta, H. Q.; Rümmeli, M. H.; Tully, C. G.; Li, Z.; Yin, W.-J.; Yang, L.; Lee, K.-B.; Yang, J.; Bozkurt, I.; Liu, S.; Zhang, W.; Chhowalla, M. Plasmon-Free SurfaceEnhanced Raman Spectroscopy Using Metallic 2D Materials. ACS Nano 2019, 13, 8312-8319.

(11) Jia, S.; Bandyopadhyay, A.; Kumar, H.; Zhang, J.; Wang, W.; Zhai, T.; Shenoy, V. B.; Lou, J. Biomolecular Sensing by SurfaceEnhanced Raman Scattering of Monolayer Janus Transition Metal Dichalcogenide. Nanoscale 2020, 12, 10723-10729.

(12) Hemmer, P. R.; Prentiss, M. G. Coupled-Pendulum Model of the Stimulated Resonance Raman Effect. J. Opt. Soc. Am. B 1988, 5, No. 1613.

(13) Hoekstra, R. M.; Telo, J. P.; Wu, Q.; Stephenson, R. M.; Nelsen, S. F.; Zink, J. I. Solvent Effects on the Coexistence of Localized and Delocalized 4,4'-Dinitrotolane Radical Anion by 
Resonance Raman Spectroscopy. J. Am. Chem. Soc. 2010, 132, 88258827.

(14) Semova, I.; Levenson, A. E.; Krawczyk, J.; Bullock, K.; Williams, K. A.; Wadwa, R. P.; Shah, A. S.; Khoury, P. R.; Kimball, T. R.; Urbina, E. M.; de Ferranti, S. D.; Bishop, F. K.; Maahs, D. M.; Dolan, L. M.; Clish, C. B.; Biddinger, S. B. Type 1 Diabetes is Associated with an Increase in Cholesterol Absorption Markers but a Decrease in Cholesterol Synthesis Markers in a Young Adult Population. J. Clin. Lipidol. 2019, 13, 940-946.

(15) Guselnikova, O.; Kalachyova, Y.; Hrobonova, K.; Trusova, M.; Barek, J.; Postnikov, P.; Svorcik, V.; Lyutakov, O. SERS Platform for Detection of Lipids and Disease Markers Prepared Using Modification of Plasmonic-Active Gold Gratings by Lipophilic Moieties. Sens. Actuators, B 2018, 265, 182-192.

(16) Wang, L.; Ge, L.; Rufford, T. H.; Zhu, Z. H. Functionalization of Carbon Nanotubes for Catalytic Applications. In Nanotubes and Nanosheets: Functionalization and Applications of Boron Nitride and Other Nanomaterials; Chen, Y., Ed.; CRC Press, 2015; pp 223-229.

(17) Samadi, M.; Sarikhani, N.; Zirak, M.; Zhang, H.; Zhang, H. L.; Moshfegh, A. Z. Group 6 Transition Metal Dichalcogenide Nanomaterials: Synthesis, Applications and Future Perspectives. Nanoscale Horiz. 2018, 3, 90-204.

(18) Fraser, J. P.; Masaityte, L.; Zhang, J.; Laing, S.; Moreno-López, J. C.; McKenzie, A. F.; McGlynn, J. C.; Panchal, V.; Graham, D.; Kazakova, O.; Pichler, T.; MacLaren, D. A.; Moran, D. A. J.; Ganin, A. Y. Selective Phase Growth and Precise-Layer Control in $\mathrm{MoTe}_{2}$. Commun. Mater. 2020, 1, No. 48.

(19) McGlynn, J. C.; Dankwort, T.; Kienle, L.; Bandeira, N. A. G.; Fraser, J. P.; Gibson, E. K.; Cascallana-Matías, I.; Kamarás, K.; Symes, M. D.; Miras, H. N.; Ganin, A. Y. The Rapid Electrochemical Activation of $\mathrm{MoTe}_{2}$ for the Hydrogen Evolution Reaction. Nat. Commun. 2019, 10, No. 4916.

(20) Song, Q.; Wang, H.; Pan, X.; Xu, X.; Wang, Y.; Li, Y.; Song, F.; Wan, X.; Ye, Y.; Dai, L. Anomalous In-Plane Anisotropic Raman Response of Monoclinic Semimetal $1 \mathrm{~T}^{\prime}-\mathrm{MoTe}_{2}$. Sci. Rep. 2017, 7, No. 17939.

(21) McGlynn, J. C.; Cascallana-Matías, I.; Fraser, J. P.; Roger, I.; McAllister, J.; Miras, H. N.; Symes, M. D.; Ganin, A. Y. Molybdenum Ditelluride Rendered into an Efficient and Stable Electrocatalyst for the Hydrogen Evolution Reaction by Polymorphic Control. Energy Technol. 2018, 6, 345-350.

(22) Wang, X.; Cao, E.; Zong, H.; Sun, M. Plasmonic Electrons Enhanced Resonance Raman Scattering (EERRS) and Electrons Enhanced Fluorescence (EEF) Spectra. Appl. Mater. Today 2018, 13, 298-302.

(23) Jones, R. R.; Hooper, D. C.; Zhang, L.; Wolverson, D.; Valev, V. K. Raman Techniques: Fundamentals and Frontiers. Nanoscale Res. Lett. 2019, 14, No. 231.

(24) Guselnikova, O.; Postnikov, P.; Marque, S. R. A.; Švorčík, V.; Lyutakov, O. Beyond Common Analytical Limits of Radicals Detection Using the Functional SERS Substrates. Sens. Actuators, B 2019, 300, No. 127015.

(25) Qiu, C.; Zhou, H.; Yang, H.; Chen, M.; Guo, Y.; Sun, L. Investigation of $n$-Layer Graphenes as Substrates for Raman Enhancement of Crystal Violet. J. Phys. Chem. C 2011, 115, 10019-10025.

(26) Ling, X.; Wu, J.; Xie, L.; Zhang, J. Graphene-ThicknessDependent Graphene-Enhanced Raman Scattering. J. Phys. Chem. C 2013, 117, 2369-2376.

(27) Lee, Y.; Kim, H.; Lee, J.; Yu, S. H.; Hwang, E.; Lee, C.; Ahn, J.H.; Cho, J. H. Enhanced Raman Scattering of Rhodamine 6G Films on Two-Dimensional Transition Metal Dichalcogenides Correlated to Photoinduced Charge Transfer. Chem. Mater. 2016, 28, 180-187.

(28) Otto, A. Theory of First Layer and Single Molecule Surface Enhanced Raman Scattering (SERS). Phys. Status Solidi A 2001, 188, $1455-1470$.

(29) Kim, J.; Jang, Y.; Kim, N.-J.; Kim, H.; Yi, G.-C.; Shin, Y.; Kim, M. H.; Yoon, S. Study of Chemical Enhancement Mechanism in Non-
Plasmonic Surface Enhanced Raman Spectroscopy (SERS). Front. Chem. 2019, 7, No. 582.

(30) Lee, H. K.; Lee, Y. H.; Koh, C. S. L.; Phan-Quang, G. C.; Han, X.; Lay, C. L.; Sim, H. Y. F.; Kao, Y. C.; An, Q.; Ling, X. Y. Designing Surface-Enhanced Raman Scattering (SERS) Platforms beyond Hotspot Engineering: Emerging Opportunities in Analyte Manipulations and Hybrid Materials. Chem. Soc. Rev. 2019, 48, 731-756.

(31) Guselnikova, O.; Postnikov, P.; Elashnikov, R.; Miliutina, E.; Svorcik, V.; Lyutakov, O. Metal-Organic Framework (MOF-5) Coated SERS Active Gold Gratings: A Platform for the Selective Detection of Organic Contaminants in Soil. Anal. Chim. Acta 2019, 1068, 70-79.

(32) Pérez León, C.; Kador, L.; Peng, B.; Thelakkat, M. Characterization of the Adsorption of Ru-Bpy Dyes on Mesoporous $\mathrm{TiO}_{2}$ Films with UV-Vis, Raman, and FTIR Spectroscopies. J. Phys. Chem. B 2006, 110, 8723-8730.

(33) Jiang, L.; You, T.; Yin, P.; Shang, Y.; Zhang, D.; Guo, L.; Yang, $S$. Surface-Enhanced Raman Scattering Spectra of Adsorbates on $\mathrm{Cu}_{2} \mathrm{O}$ Nanospheres: Charge-Transfer and Electromagnetic Enhancement. Nanoscale 2013, 5, 2784-2789.

(34) Kurban, S.; Erkoç, F.; Erkoç, Ş. Quantum Chemical Treatment of $\beta$-Sitosterol Molecule. Pharm. Biol. 2010, 48, 637-642.

(35) McGlynn, J. C.; Friskey, M.; Ganin, A. Y. Parameter Optimisation for Electrochemically Activated $\mathrm{MoTe}_{2}$. Sustainable Energy Fuels 2020, 4, 4473-4477.

(36) International Conference on Harmonisation of Technical Requirements for Registration of Pharmaceuticals for Human Use ICH Harmonised Tripartite Guidline Validation of Analytical Procedures: Text and Methodology Q2(R1).

(37) Guselnikova, O.; Samant, R.; Postnikov, P.; Trelin, A.; Svorcik, V.; Lyutakov, O. Plasmon-Assisted Self-Cleaning Sensor for the Detection of Organosulfur Compounds in Fuels. J. Mater. Chem. C 2019, 7, 14181-14187.

(38) Fu, X.; Chu, Y.; Zhao, K.; Li, J.; Deng, A. Ultrasensitive Detection of the $\beta$-Adrenergic Agonist Brombuterol by a SERS-Based Lateral Flow Immunochromatographic Assay Using Flower-like GoldSilver Core-Shell Nanoparticles. Microchim. Acta 2017, 184, 17111719.

(39) Kalachyova, Y.; Mares, D.; Jerabek, V.; Elashnikov, R.; Švorčík, V.; Lyutakov, O. Longtime Stability of Silver-Based SERS Substrate in the Environment and (Bio)Environment with Variable Temperature and Humidity. Sens. Actuators, A 2019, 285, 566-572.

(40) Dumont, E.; De Bleye, C.; Cailletaud, J.; Sacré, P. Y.; Van Lerberghe, P. B.; Rogister, B.; Rance, G. A.; Aylott, J. W.; Hubert, P.; Ziemons, E. Development of a SERS Strategy to Overcome the Nanoparticle Stabilisation Effect in Serum-Containing Samples: Application to the Quantification of Dopamine in the Culture Medium of PC-12 Cells. Talanta 2018, 186, 8-16.

(41) Sun, F.; Zhang, P.; Bai, T.; Galvan, D.; Hung, H. C.; Zhou, N.; Jiang, S.; Yu, Q. Functionalized Plasmonic Nanostructure Arrays for Direct and Accurate Mapping Extracellular PH of Living Cells in Complex Media Using SERS. Biosens. Bioelectron. 2015, 73, 202-207.

(42) Wang, X.; Huang, S. C.; Hu, S.; Yan, S.; Ren, B. Fundamental Understanding and Applications of Plasmon-Enhanced Raman Spectroscopy. Nat. Rev. Phys. 2020, 2, 253-271.

(43) Jensen, L.; Aikens, C. M.; Schatz, G. C. Electronic Structure Methods for Studying Surface-Enhanced Raman Scattering. Chem. Soc. Rev. 2008, 37, 1061-1073.

(44) Morton, S. M.; Jensen, L. Understanding the Molecule-Surface Chemical Coupling in SERS. J. Am. Chem. Soc. 2009, 131, 40904098.

(45) Lippitsch, M. E. Ground-State Charge Transfer as a Mechanism for Surface-Enhanced Raman Scattering. Phys. Rev. B 1984, 29, No. 3101.

(46) Computational Chemistry Comparison and Benchmark DataBase Release 21 (August 2020), Standard Reference Database 101, National Institute of Standards and Technology, CCCBDB List of Experimental Polarizabilites. https://cccbdb.nist.gov/pollistx.asp. 
(47) Chen, R.; Jensen, L. Interpreting the Chemical Mechanism in SERS Using a Raman Bond Model. J. Chem. Phys. 2020, 152, No. 024126.

(48) Furtak, T. E.; Roy, D. Nature of the Active Site in SurfaceEnhanced Raman Scattering. Phys. Rev. Lett. 1983, 50, No. 1301.

(49) Granovsky, A. A. Firefly Version 8.0.0. http:// classic.chem.msu. su/gran/firefly/index.html, accessed 10.102020.

(50) Casida, M. E.; Chong, D. P. Recent Advances in Density Functional Methods-Part 1; Chong, D. P., Ed.; World Scientific: Singapore, 1995; pp 155-192.

(51) Lee, C.; Yang, W.; Parr, R. G. Development of the ColleSalvetti Correlation-Energy Formula into a Functional of the Electron Density. Phys. Rev. B 1988, 37, No. 785.

(52) Rassolov, V. A.; Ratner, M. A.; Pople, J. A.; Redfern, P. C.; Curtiss, L. A. 6-31G* Basis Set for Third-Row Atoms. J. Comput. Chem. 2001, 22, 976-984.

(53) https://www.chemcraftprog.com, accessed 10.102020. 